\title{
Peroperative microbial contamination of anterior chamber aspirates during extracapsular cataract extraction and phacoemulsification
}

Bijan Beigi, William Westlake, Else Mangelschots, Bernard Chang, Walter Rich, Terry Riordan

\begin{abstract}
Background-The normal conjunctival flora is one of the main sources of intraocular contamination during cataract surgery. The theory that the positive anterior chamber (AC) pressure during phacoemulsification (phaco), and the smaller wound utilised, might reduce the rate of contamination was studied.

Methods-The peroperative AC aspirates of 210 consecutive patients undergoing cataract surgery were assessed. In group 1, 100 patients underwent a standard extracapsular cataract extraction (ECCE). In group 2, 110 patients underwent phacoemulsification of the crystalline lens through a scleral tunnel. AC aspirates from the Simcoe irrigation/ aspiration cannula (group 1) and phaco probe (group 2) were collected and microbiological studies performed after direct and enrichment cultures.

Results-There were 29 (29\%) positives in the ECCE group compared with $22(20 \%)$ positive cultures from $\mathrm{AC}$ aspirates in the phaco group. Coagulase negative staphylococcus (CNS) was the commonest contaminant in both groups.

Conclusion-Although there was a higher rate of AC contamination during ECCE, the difference was not statistically significant $\left(\mathrm{p}>0.10, \chi^{2}=2.31\right)$.

(Br F Ophthalmol 1997;81:953-955)
\end{abstract}

The overwhelming majority of postoperative intraocular infections are caused by an organism that is introduced at the time of the surgery. The major source of intraocular contamination is the conjunctival flora. Organisms enter the anterior chamber (AC) either directly ${ }^{1}$ or indirectly by intraocular lenses. ${ }^{2}$ The role of such contamination in the production of postoperative inflammation and infection is well recognised. ${ }^{34}$ It would therefore seem prudent to minimise bacterial entry into the AC during cataract surgery to reduce the risk of postoperative endophthalmitis. Careful preoperative preparation can greatly influence the rate of potential microbial contaminants. Attention to surgical technique can further reduce the intraocular delivery of microorganisms. For example, an AC maintained at higher than atmospheric pressure might have a lower rate of bacterial contamination.
Theoretically, the number of microorganisms entering the AC peroperatively might be reduced in phacoemulsification surgery, because of the constant infusion of fluid at greater than atmospheric pressure and the smaller incision. The aim of this study was to assess the effect of two different techniques of cataract extraction - extracapsular cataract extraction (ECCE) and phacoemulsification (phaco), on the rate of AC microbial contamination.

\section{Material and methods}

Peroperative anterior chamber aspirates obtained from 210 consecutive patients undergoing routine uncomplicated cataract extraction with posterior chamber intraocular lens (PCIOL) were examined. None had any active external eye or general infectious disease. Topical or oral antibiotics were not used preand peroperatively.

One hundred consecutive patients had a standard ECCE with PCIOL (group 1). One hundred and ten patients had phaco with PCIOL (group 2). One hour preoperatively the pupil was dilated with cyclopentolate $1 \%$ and phenylephrine $2.5 \%$. The eye was anaesthetised using a peribulbar block with $10 \mathrm{ml} \mathrm{mix-}$ ture containing equal parts bupivacaine $0.5 \%$ and lignocaine $2 \%$ with $300-1500$ IU hyaluronidase.

The skin of the eyelids and conjunctival sac was cleaned with povidone-iodine $10 \%$ and $5 \%$ respectively. A sterile drape with an adhesive foil was used to remove the eyelashes from the operative field. Irrigation was provided from a gravity feed bottle of Hartmann's or balanced salt solution (BSS) containing 0.6 $\mathrm{ml}$ adrenaline $1 / 1000$ per $500 \mathrm{ml}$. The infusion tubing did not contain a bacterial filter.

In group 1 a standard ECCE with PCIOL was performed using a limbal stepped section. The crystalline lens was then expressed and the remaining soft lens matter removed using a Simcoe irrigation/aspiration cannula. The syringes containing the $\mathrm{AC}$ aspirates were retained for culture. A PCIOL was then inserted in the posterior chamber and the posterior limbal wound was sutured with $10 / 0$ nylon sutures and covered with conjunctiva.

In group 2 phaco was performed through a 3 $\mathrm{mm}$ scleral tunnel using a frown incision. A 1.5 $\mathrm{mm}$ corneal paracentesis was made for insertion of the second instrument. Continuous circular capsulorrhexis was completed under vis-
Accepted for publication 4 June 1997 
Table 1 Results of cultures obtained from group 1 (ECCEs) (sample size $=100$, negative cultures $=71$, positive $=29(29 \%))$

Number of bacterial species cultured from anterior chamber aspirates

\begin{tabular}{lllll}
\cline { 2 - 5 } Type of culture & Positive & 1 type & 2 types & 3 types \\
\hline Direct & 2 & 1 & 0 & 1 \\
Enrichment & 29 & 15 & 12 & 2 \\
\hline
\end{tabular}

coelastic and the crystalline lens then extracted using the divide and conquer technique. The cortex was removed by automated irrigation/ aspiration probe. The wound was extended to $5.5 \mathrm{~mm}$ and a one piece PMMA $5 \times 6 \mathrm{~mm}$ intraocular lens (Iolab) inserted in the bag. The self sealing scleral wound was covered with conjunctiva. The phaco probe aspirates were poured into a sterile specimen bottle from the collection cassette and sent for culture. Fifty control specimen bottles containing sterile sodium chloride were sent among the study specimens.

\section{MICROBIOLOGICAL STUDIES}

The aspirates collected during ECCE and phaco were treated identically. A volume of 0.1 $\mathrm{ml}$ of fluid was inoculated onto three plates $-5 \%$ blood agar, chocolate agar, and Sabouraud agar. The chocolate agar was incubated at $37^{\circ} \mathrm{C}$ in $5 \%$ carbon dioxide for 48 hours. The blood agar was incubated in an anaerobic cabinet for a total of 7 days and the Sabouraud plate at $37^{\circ} \mathrm{C}$ for 7 days aerobically.

In addition the fluid was centrifuged and the deposit resuspended in $2 \mathrm{ml}$ of fluid. This was then inoculated into Pedi-bact blood culture broth (Organon-Technika) supplemented with $1 \mathrm{ml}$ of Fildes extract. The bottles were incubated in the BacTalert machine for 7 days.

Any bottles flagging positive were unloaded and subcultured aerobically and anaerobically. Any positive growth, even if scanty, was recorded and isolates were identified to genes/ species level using standard methods.

\section{STATISTICAL ANALYSIS}

The $\chi^{2}$ test was used to compare the results in the two groups. We calculated that our sample sizes would have an $80 \%$ chance of producing

Table 2 Results of AC cultures obtained from group 2 (phaco) (sample size $=110$, negative $=88$, positive $=22$ $(21 \%))$

\begin{tabular}{lllll}
\hline & \multicolumn{2}{l}{$\begin{array}{l}\text { Number of bacterial species cultured from } \\
\text { anterior chamber aspirates }\end{array}$} \\
\cline { 2 - 5 } Type of culture & Positive & 1 type & 2 types & types 3 \\
\hline Direct & 6 & 4 & 2 & - \\
Enrichment & 21 & 19 & 2 & - \\
\hline
\end{tabular}

a statistically significant result at $\mathrm{p}<0.05$ (EPI INFO Stat Calc).

\section{Results}

In group 1 (ECCE), bacteria were cultured from AC aspirates in 29 cases (29\%). There were two positive direct and 29 positive enrichment cultures (Table 1) which included; 32 coagulase negative staphylococcus (CNS), five Staphylococcus aureus, five diphtheroids, two Streptococcus viridans, and one Moraxella.

In group 2 (phaco), bacteria were cultured from AC aspirates of 22 cases (20\%). Six were from direct and 21 from enrichment cultures (Table 2). Enrichment cultures included; 15 CNS, two a haemolytic Streptococcus, two Acinetobacter species, one $\beta$ haemolytic Streptococcus, one Moraxella, one Candida, and one non-fermentive oxidase positive Gram negative rod.

None of the 50 control bottles cultured positive. Although there was a lower rate of contamination of the AC aspirates during phaco, this difference between the two groups was not statistically significant $\left(\chi^{2}=2.31\right.$ : $\mathrm{p}=0.129)$.

\section{Discussion}

Despite technical improvements in the field of intraocular surgery and all the precautions taken, endophthalmitis is still a major postoperative complication. The reported incidence following ECCE is about $0.1 \%,{ }^{5}$ ranging between $0.05 \%$ and $0.3 \% .{ }^{6}$ However, the true figure is likely to be higher than this, many milder cases being both unrecognised and unreported and considered as sterile postoperative inflammation. The normal flora of the ocular surface and conjunctiva, especially CNS, is well known to produce postoperative inflammation and infections ${ }^{13}$ and is thought to be the main source of contamination. Irrigation of the conjunctiva before and during surgery introduces more micro-organisms from the conjunctival crypts onto the surface. ${ }^{7}$ Airborne microbes can also be delivered into the AC by means of intraocular lenses. ${ }^{2}$

It is accepted that preoperative use of povidone-iodine reduces the microbial contamination of the operating field significantly ${ }^{8} 9$ and was used in this study. Preoperative antibiotics were not used since they have only a limited effect on reducing ocular surface bacteria. $^{10}$

Surgical techniques can alter the delivery rate of microbes into the $\mathrm{AC}$ and vitreous cavity. The incidence of endophthalmitis is higher following intracapsular cataract extraction ${ }^{5}$ when compared with ECCE. It is also higher in the event of a capsular rupture. ${ }^{11}$ Anterior

Table 3 Published results comparing AC contamination of phaco and ECCE

\begin{tabular}{|c|c|c|c|c|c|c|c|c|}
\hline & \multicolumn{3}{|c|}{ Sample size } & \multicolumn{2}{|c|}{ Positive cultures } & \multicolumn{2}{|c|}{ Negative cultures } & \multirow[b]{2}{*}{$p$ Value $\left(\chi^{2}\right)$} \\
\hline & Phaco & $E C C E$ & Total & Phaco & $E C C E$ & Phaco & $E C C E$ & \\
\hline Current study & 110 & 100 & 210 & $22(20 \%)$ & $29(29 \%)$ & $88(80 \%)$ & $71(71 \%)$ & 0.129 \\
\hline Manners et $a l^{15}$ & 50 & 50 & 100 & $10(20 \%)$ & $12(24 \%)$ & $40(80 \%)$ & $38(76 \%)$ & 0.629 \\
\hline Egger $e t a l^{12} 13$ & 26 & 174 & 200 & $2(7.6 \%)$ & $49(28 \%)$ & $24(92.4 \%)$ & $125(72 \%)$ & 0.026 \\
\hline Total & 186 & 324 & 510 & $34(18.3 \%)$ & $90(27.8 \%)$ & $152(81.7 \%)$ & $234(72.2 \%)$ & 0.016 \\
\hline
\end{tabular}


chamber collapse and shallowing during the aspiration phase of ECCE can introduce microbes into the AC owing to a pressure lower than the atmosphere. ${ }^{2}$ This has been the basis for speculation that phaco with the use of a small incision and a better preservation of the AC throughout the surgery can reduce the rate of contamination.

Egger et al in two identical studies of 200 patients undergoing cataract extractions have shown less intraocular contamination with phaco. $^{12} 13$ The AC aspirates of 173 patients undergoing ECCE were compared with only 26 cases of phaco (Table 3). The study was rather weak because of the low numbers in the phaco group, although the results did reach statistical significance. Their conclusion was quoted in a subsequent editorial ${ }^{14}$ and in support of a similar claim.

Manners et al compared two groups of 50 patients and reported $20 \%$ and $24 \%$ contamination during phaco and ECCE respectively. The difference was not statistically significant. ${ }^{15}$

We are not aware of larger studies to assess the effect of different surgical techniques on peroperative intraocular microbial contamination during cataract surgery. In this study the AC contamination during phaco $(20 \%)$ was less than ECCE (29\%), but this was not statistically significant $(p>0.1)$. However, for a similar rate of positive cultures around 200 patients would be required in each group to achieve statistical significance $(\mathrm{p}<0.05)$. A metaanalysis on the data so far reported (Table 3) also supports the assertion that there is a reduced bacterial contamination of the AC during phaco $\left(\chi^{2}=5.79: \mathrm{p}<0.05\right)$

Of the 58 positive cultures, 50 were obtained using enrichment techniques. There were only six positive direct cultures in phaco and two in the ECCE group (no statistical significance). Enrichment cultures reduce the chance of false negative cultures (Tables 1 and 2) and increase the likely detection of a low level of contamination. It is possible that cases with low levels of contamination produce a subclinical endophthalmitis and mild postoperative uveitis, ${ }^{24}$ though this requires further investigation.

\section{Conclusion}

Although open to question, to minimise the risk of postoperative endophthalmitis, the number of bacteria left in situ should be reduced to a minimum. ${ }^{16}$ Proper preoperative preparation of the eye and adherence to standard sterilisation protocols are important. The use of peroperative intracameral antibiotics has also been advocated. ${ }^{17}$
An uncomplicated, well controlled surgical technique is desirable. Small incision cataract extraction using phaco is ideally a controlled procedure that facilitates the maintenance of a well formed AC throughout the operation and is probably associated with less peroperative bacterial influx. In this study we have been unable to prove that the lower rate of bacterial contamination of the AC during phaco was not due to chance alone.

We have found enrichment cultures valuable with a much higher sensitivity to identify bacteria. Their use, especially for detection of low grade infection, seems to minimise the rate of false negative results.

The authors would like to thank all the theatre staff in the West of England Eye Unit and Microbiology Department, particularly Mr L Joslin, for their contribution. We are also grateful for Mr A Alaghebandian's help and support.

1 Sherwood DR, Rich WJ, Jacob JC, Hart RJ, Fairchild YL. Bacterial contamination of intraocular and extraocular fluids during extracapsular cataract extraction. Eye 1989;3:308-12.

2 Doyle A, Beigi B, Early A, Blake A, Eustace P, Hone R. Adherence of bacteria to intraocular lenses, a prospective study. Br f Ophthalmol 1995;79:347-9.

3 Piest KL, Kincaid MC, Tetz MR, Apple DJ, Roberts WA, Price FW, Jr. Localized endophthalmitis. A newly described Price FW, Jr. Localized endophthalmitis. A newly described
cause of the so-called toxic lens syndrome. F Cataract cause of the so-called toxic
Refract Surg 1987;13:498-510

4 Meisler DM, Palestine AG, Vastine DW, Demartin DR, Murphy BF, Reinhart WJ, et al. Chronic propionebacter endophthalmitis. Am $\mathcal{F}$ Ophthalmol 1986;102:733-9.

5 Javitt JC, Vitale S, Canner JK, Street DA, Krakauer H, McBean AM, Sommer A. National outcomes of cataract extraction. Arch Ophthalmol 1991;109:1085-9.

6 Kattan HM, Flynn HW Jr, Pflugfelder SC, Robertson C, Forster RK. Nosocomial endophthalmitis survey:current incidence of infection after intraocular surgery. Ophthalmology 1991;98:227-38.

7 Isenberg SJ, Apt L, Yoshimuri R. Chemical preparation of the eye in ophthalmic surgery. Effect of conjunctival irrigation. Arch Ophthalmol 1983;101:761-3.

8 Mork P. Polyvinylpyrrolidone-iodine as a disinfectant in eye surgery for five years. Acta Ophthalmol 1987;65:572-4.

9 Isenberg SJ, Apt L, Yoshimuri R, Khwarg S. Chemical isenberg SJ, Apt L, Yoshimuri R, Khwarg S. Chemical
preparation of the eye in ophthalmic surgery. Arch Ophthalmol 1985; 103:1340-2.

10 Chitkara DK, Manners T, Chapman F, Stoddant Mg, Hill D, Jenkins D. Lack of effect of the preoperative norfloxacin on bacterial contamination of anterior chamber aspirates after cataract surgery. Br f Ophthalmol 1994;78:772-4

1 Jager GV, Brinkman CJ, Van Tilburg CJ, Beekhuis WH, Joosse MV. Pseudophakic endophthalmitis. Doc Ophthalmol 1992;82:109-14.

12 Egger SF, Huber-Spitzy V, Scholda C, Schneider B, Grabner G. Bacterial contamination during extracapsular cataract extraction. Ophthalmologica 1994;208:77-81.

13 Egger SF, Huber-Spitzy V, Skorpik C, Weghaupt H, Schloda $\mathrm{C}$, Arocker-Mettinger E, et al. Different techniques of extrapcapsular cataract extraction, bacterial contamination during surgery. Graefes Arch Clin Exp Ophthalmol 1994;232: during sur

14 Towler H. Aqueous contamination during small incision cataract surgery:a lesson in study design. Br $\mathcal{F}$ Ophthalmol 1995;79:873.

15 Manners TD, Chitkara DK, Marsh PJ, Stoddart MG. Anterior chamber aspirate cultures in small incision cataract surgery. Br f Ophthalmol 1995;79:878-80.

16 Foster RK, Zachery IG, Cottingham AJ, Norton EWD. Further observation on the diagnosis, cause and treatment of endophthalmitis. Am f Ophthalmol 1976;81:52-6.

17 Gimbel HV, De Brof RS, De Brof BM. Prophylactic intracameral antibiotics during cataract surgery: the incidence of endophthalmitis and corneal endothelial cell loss. Eur $\mathcal{f}$ Refract Surg 1994;6:280-5. 